\title{
Ballistocardiographic artifacts in PPG imaging
}

\author{
Andreia V. Moço, Sander Stuijk, and Gerard de Haan
}

\begin{abstract}
Objective: Photoplethysmography (PPG) is a noninvasive technique to measure the blood-volume pulse and derive various vital signs. Camera-based PPG imaging was recently proposed for clinical microvascular assessment, but motion robustness is still an issue for this technique. Our study aims to quantify cardiac-related, i.e., ballistocardiographic (BCG), motion as a source of artifacts in PPG imaging. Methods: In this paper, using the human head as a relevant region of interest, the amplitude of BCG-artifacts was modeled for a Lambertian surface illuminated by a light source. To derive peak-to-peak head displacements for the model, we recorded, on 54 subjects, PPG and inertial sensor data at the pulse and cranial vertex. We simulated the effect of light source location at a mesh representation of a human face and conducted additional experiments on a real subject. Results: Under non-orthogonal illumination, the relative strength of $\mathrm{BCG}$-artifacts is strong enough, compared to the amplitude of PPG signals, to compromise PPG imaging in realistic scenarios. Particularly affected are the signals obtained in the non-green part of the spectrum and/or when the incident angle at the skin surface exceeds 45 degrees. Conclusion: From the model and an additional experiment conducted on real skin, we were able to prove that homogenous and orthogonal illumination is a means to minimize the problem. Significance: Our illumination recommendation provides a simple and effective means to improve the validity of remote PPG-imagers. We hope that it helps to prevent mistakes currently seen in many publications on remote PPG.
\end{abstract}

Index Terms-PPG imaging, ballistocardiography, artifacts.

\section{INTRODUCTION}

Photoplethysmography (PPG) is a non-invasive optical technique to detect blood volume changes in the microvascular bed of tissue. Since transient changes to the cardiovascular blood pulse-volume modulate incident light, a PPG waveform may be obtained by illuminating the skin and measuring the amount of light reflected, or transmitted, to a photosensor. The thusobtained waveform is a rich signal that provides information on cardiac activity, respiration, $\mathrm{S}_{p} \mathrm{O}_{2}$, vasomotor activity and thermal regulation. PPG signals are now widely used in hospitals [1]. However, typical PPG systems require sensors to be in direct contact with the skin, which is a drawback, particularly for continuous long-term monitoring or frequently repeated measurements. In specific populations with damaged or highly sensitive skin (e.g., burned patients, the elderly and newborns) the application of contact based monitoring may

A. Moço with the Electronic Systems Group, Eindhoven University of Technology, Eindhoven, The Netherlands, e-mail: (A.v.Moco@tue.nl).

S. Stuijk is with the Electronic Systems Group, Eindhoven University of Technology, Eindhoven, The Netherlands, e-mail: (S.Stuijk@tue.nl).

G. de Haan is with the Philips Innovation Group, Philips Research, Eindhoven 5612 AZ, The Netherlands, e-mail: (G.de.Haan@Philips.com)

Manuscript received June, 20, 2015; revised August, 23, 2015 and October, 9, 2015; accepted November, 14, 2015.

Copyright (c) 2015 IEEE. Personal use of this material is permitted However, permission to use this material for any other purposes must be obtained from the IEEE by sending an email to pubs-permissions@ieee.org. even be impossible. However, if PPG signals are extracted remotely, such issues can be solved in an unobtrusive, userfriendly and low-cost manner.

Pioneering remote PPG (PPG) measurement protocols described in early papers required dedicated equipment [2], [3]. However, the feasibility of PPG measurements using ambient light and consumer electronics RGB cameras was also demonstrated [4], as well as using thermal [5] and infrared cameras [6]. Efforts have also been made to apply remote monitoring to microvascular research by extending the PPG technique to PPG imaging (PPGI) [7]. Remote PPG imagers are computer-based CCD systems that non-invasively visualize local changes in dermal blood volume using backscattered optical radiation [8]. Recent advances in PPGI allow mapping of blood perfusion in static subjects and hold the promise of application in anesthetized patients [9]. However, enthusiasm about PPGI is tempered by the immature state of the technology, in particular when it comes to motion robustness.

In this paper, we focus on quantifying a particular motion threat to PPGI that has so far received little attention in the literature. In general, motion with respect to the light source modulates light [10]. Hence, an PPG-camera captures the summed effect of motion and changes in blood volume (PPG). Band-pass filters and correlation-based approaches are typically used to reduce motion components in PPG images [11], but these have little effect if motion itself is related to the cardiac activity. This effect of pulsatile activity is referred to in the literature as ballistocardiographic motion.

Ballistocardiography (BCG) was almost forgotten for some time due to development of high resolution electrochocardiography and modern methods of imaging [12]. However, recently it comes back into favor as a complementary method. For example, the latest literature shows both conventional electronic BCG systems designed for home use [13] as well as fiber-optic sensors for monitoring MRI patients [14]. Although of small amplitude, there is compelling evidence for the detectability of BCG signals at the thorax [15] and also head, either using inertial sensors [16], [17], cameras [18], or simultaneous electroencephalography-fMRI [14], [19].

To our knowledge, only Blanik et al. [20] and Verkruysse et al. [4] reported movement artifacts resulting from heartbeating in functional mappings. However, the description of this effect was restricted to selected skin sites (edges of the forearm, carotid artery) and the relation between the amplitude of BCG-artifacts and lighting conditions was not investigated. Accordingly, the aim of this investigation is to quantify the relative effect of cardiac-induced motion on the measured PPG-image. In a first stage, we model the effect of motion on the reflected light. Then, we assess implications for PPGI by apply the model for various lighting conditions, using the face as a relevant measurement site. Finally, we provide a 
proof-of-concept experiment on real skin.

\section{MATERiAls AND METHODS}

Section II-A describes the working scenario and models the relation between micro-motion and the resulting BCGartifacts for a Lambertian surface illuminated by a punctual light source. Section II-B presents the methodology for modeling BCG-artifacts at a mesh representation of a human face, for different illumination settings. Finally, Section II-C demonstrates actual BCG effects on the human forehead.

\section{A. A model for BCG-artifacts at the head}

1) Scenario and assumptions: Fig. 1 depicts a headmounted device for simultaneous acquisition of BCG-motion and artifact signals at the subject's cranial vertex (most superior point on the skull [21]), where a motion sensor (MS) is covered by a white and opaque board similar to spectralon, providing an horizontal surface for video recording. In this sense, BCG-artifacts are subtle brightness variations, recorded over time, by a static camera pointing to a RoI in the surface. Since the MS and the surface are attached together, they share the same referential axis.

We consider a punctual light source located in the $Y_{0} Z$ plane, at a distance $R$ from a small RoI in the board surface. Assuming uniform illumination, the direction of light at any point of the RoI is given by the unit vector $\hat{\mathbf{e}}_{\mathbf{r}}=$ $(0, \sin \alpha,-\cos \alpha)^{T}$, oriented outward from the source, where $\alpha$ is the incident angle between light source and RoI.

For the sake of simplicity, we tackle the scenario of seated subjects that are not performing voluntary motions. We consider that the head-neck system is confined to rotation around the first cervical vertebra [22] and translation due to respiration or heart beating. BCG-motion was quantified as linear displacements $\left(d_{X}, d_{Y}, d_{Z}\right.$, for the $X, Y$ and $Z$ axis) and rotation ( $\theta_{X}$, extension/flexion; $\theta_{Y}$, lateral bending; $\theta_{Z}$, axial rotation). The sign of the angles is in agreement to Euler's rotation theorem (positive for counterclockwise rotation). Accordingly, micro-motion modulates $\hat{\mathbf{e}}_{\mathbf{n}}(t)$ as follows:

$$
\hat{\mathbf{e}}_{\mathbf{n}}(t)=\mathbf{R}_{Z}\left(\theta_{Z}(t)\right) \mathbf{R}_{Y}\left(\theta_{Y}(t)\right) \mathbf{R}_{X}\left(\theta_{X}(t)\right) \hat{\mathbf{e}}_{\mathbf{n}, \mathbf{0}},
$$

where $\hat{\mathbf{e}}_{\mathbf{n}, \mathbf{0}}=(0,0,1)^{T}$ is the baseline normal vector to the surface and $\mathbf{R}_{X}, \mathbf{R}_{Y}$ and $\mathbf{R}_{Z}$ are the rotation matrixes about the $X, Y$ and $Z$ axis, respectively. For convenience, hereafter we will omit the explicit time dependence $(t)$ of all signals. The components of $\hat{\mathbf{e}}_{\mathbf{n}}$ are as displayed in (2).

$$
\left\{\begin{array}{l}
e_{n, X}=\cos \theta_{Z} \sin \theta_{Y} \cos \theta_{X}+\sin \theta_{Z} \sin \theta_{X} \\
e_{n, Y}=\sin \theta_{Z} \sin \theta_{Y} \cos \theta_{X}-\sin \theta_{X} \cos \theta_{Z} \\
e_{n, Z}=\cos \theta_{Y} \cos \theta_{X}
\end{array}\right.
$$

Recognizing that $\theta_{A X I S}$ is small, the Taylor-series expansion is applicable to (2). Replacing $\sin \left(\theta_{A X I S}\right) \approx \theta_{A X I S}$ and $\cos \left(\theta_{A X I S}\right) \approx 1$ into the components of $\hat{\mathbf{e}}_{\mathbf{n}}$ yields

$$
\left\{\begin{array}{l}
e_{n, X}=\theta_{Y}+\theta_{Z} \theta_{X} \\
e_{n, Y}=\theta_{Z} \theta_{Y}-\theta_{X} \\
e_{n, Z}=1
\end{array}\right.
$$

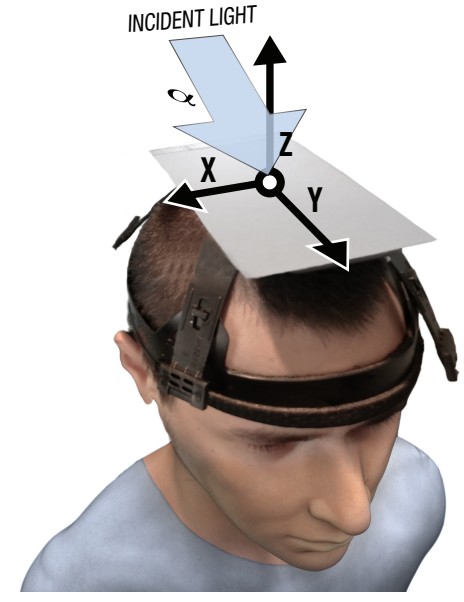

Fig. 1. Reference axis and head-mounted device for simultaneous acquisition of BCG-motion and artifact signals. The image plane contains a surface attached to the subject's head ( $X_{0} Y$ plane). The incident light rays are parallel to the $Y_{0} Z$ plane and make an angle $\alpha$ w.r.t. the surface.

2) BCG-artifacts resulting from head motion: We now model the average light intensity at an RoI in the surface, $I$, taking motion and light source location (incident angle, $\alpha$, and distance to surface, $R$ ) into account. Using the Inverse Square Law, $I$ is expressed as

$$
I=\frac{I_{0}}{\left(R+\delta_{\theta, d}\right)^{2}}\left\|\hat{\mathbf{e}}_{\mathbf{r}} \cdot \hat{\mathbf{e}}_{\mathbf{n}}\right\|,
$$

where $I_{0}$ is a constant and $\delta_{\theta, d}$ is a perturbation due to BCG movements. We proceed by considering a distant light source. For this case, since $\left(R+\delta_{\theta, d}\right)^{2} \approx R^{2}$, the only mechanism by which BCG-motion modulate $I$ is the inner product $\hat{\mathbf{e}}_{\mathbf{r}}$. $\hat{\mathbf{e}}_{\mathbf{n}}$, which is constant over the RoI. This scenario can even be simplified further by recognizing that, since the surface is uniform and horizontal, translation movements about the $X_{0} Y$ plane have no influence over brightness values recorded by a camera pointing to a static RoI. Accordingly, the average brightness at a $R O I$ in the surface, $I$, is expressed as

$$
I=\left(-e_{n, Y} \sin \alpha+e_{n, Z} \cos \alpha\right) \cdot \frac{I_{0}}{R^{2}} .
$$

For the general case of rotation in all axis of rotation, the substitution of the approximations $e_{n, Y} \approx \theta_{Z} \theta_{Y}-\theta_{X}$ and $e_{n, Z} \approx 1$ into (5) results in

$$
I \approx\left[-\left(\theta_{Z} \theta_{Y}-\theta_{X}\right) \sin \alpha+\cos \alpha\right] \cdot \frac{I_{0}}{R^{2}} .
$$

Equation (6) can be approximated recognizing that, for small angles, the mixed product term $\theta_{Z} \theta_{Y}$ is much smaller than $\theta_{X}$. Neglecting $\theta_{Z} \theta_{Y}$ is further supported by evidence for $\theta_{X}$ being stronger than either $\theta_{Z}$ or $\theta_{Y}$ [19], [17], as well as preliminary results from this study, pointing to maximal peakto-peak $(\mathrm{P} 2 \mathrm{P})$ displacements for flexion / extension $\left(\approx 0.1^{\circ}\right)$. Therefore,

$$
I \approx\left[\theta_{X} \sin \alpha+\cos \alpha\right] \cdot \frac{I_{0}}{R^{2}} .
$$

It is apparent from (7) that flexion and extension movements of the neck, $\theta_{X}$, have the strongest modulating effect over $I$. 
Also, we can recognize a time-varying ("AC") and a constant ("DC") component in $I$ :

$$
\begin{aligned}
& I_{A C} \approx \theta_{X} \sin \alpha \cdot I_{0} / R^{2} \\
& I_{D C} \approx \cos (\alpha) \cdot I_{0} / R^{2}
\end{aligned}
$$

suggesting the ratio $B C G=I_{A C} / I_{D C}$ as a convenient means of expressing the relative BCG-artifact signal:

$$
B C G \approx \frac{\theta_{X} \sin (\alpha)}{\cos (\alpha)}
$$

Equation (10) is of the kind $y \approx m . \theta_{X}$, where the slope, $m=\tan (\alpha)$, is a factor amplifying head rotation.

Let us now examine the amplitude of BCG-artifacts when the light source is closer to the surface. For this case, if the RoI is small enough for the assumption of uniform illumination to hold, $I$ is still expressed as in (4), but $\hat{\mathbf{e}}_{\mathbf{r}}$ is no longer the same for the baseline and displaced positions of the RoI. Instead, it can be shown that this vector is, approximately, $(0, \sin \alpha,-\cos \alpha+\Delta z / R)^{T}$, where $\Delta z$ denotes the displacement of the centroid of the RoI in the $Z$ axis. Using (2) and Taylor approximations, it can be further shown that $\Delta z \approx \rho\left(\theta_{X}^{2}+\theta_{Y}^{2}\right) / 2$, where $\rho$ is the distance between RoI and axis of rotation of the head-neck system. For adult subjects, $\rho$ is estimated to be within the range $15-20 \mathrm{~cm}$ and $R$ is typically larger than $30 \mathrm{~cm}$. Thus, it remains safe to ignore the contribution of $\Delta z$ to $\hat{\mathbf{e}}_{\mathrm{r}}$ and that of $\delta_{\theta, d}=\Delta z^{2}$ (see (4)), so the insights derived from distant light sources hold.

3) Experimental verification of BCG-artifacts: Figure 2 illustrates an experimental setup to verify (10) by simultaneous acquisition of motion and artifact signals. For this verification, two contrasting scenarios (i.e., near-orthogonal vs. near-frontal) seem to be appropriate ${ }^{1}$, so experiments were conducted for the incident angles, $\alpha$, set to $25^{\circ}$ and $80^{\circ}$. Head motion was quantified using accelerometry and gyroscopy, whereas pulse oximetry was used for identifying cardiac cycles and estimating instantaneous heart-rate. Data acquisition and signal processing were as follows.

Data acquisition: A subject was asked to sit on a rigid chair with back-support and wear a motion sensor (referential axis as in Fig. 1). This device was attached to his cranial vertex using adjustable straps. Because BCG waveforms are typically low-amplitude, the sensitivity and sampling rate of the motion sensors are critical parameters. As such, the chosen model was MP67B (InvenSense Inc., USA) embedded on an iPhone 6 platform (Apple Inc., USA), owing to its 16-bit resolution, low noise (gyroscope: $0.01^{\circ} / \mathrm{s} / \sqrt{\mathrm{Hz}}$, accelerometer: $0.3 \mathrm{mg} / \sqrt{\mathrm{Hz}}$ ) and possibility to sample signals at $100 \mathrm{~Hz}$. To minimize noncardiac movements, explicit instructions were given to avoid voluntary hand and facial movements during recordings. The

\footnotetext{
${ }^{1}$ Although it would be possible to verify the model for additional/alternative incident angles, these would come at the cost of increased measurement time (i.e., discomfort for the subject), as well as a more complicated setup with additional technical difficulties; for example, when the camera is frontal to the plastic board surface, orthogonal illumination $\left(0^{\circ}\right)$ would result into stronger specular reflections. Moreover, propagation of measurement errors would make it difficult to differentiate results for the incident angles of $0^{\circ}$ and $25^{\circ}$. Likewise, for $90^{\circ},(10)$ is undefined (asymptotic behavior).
}

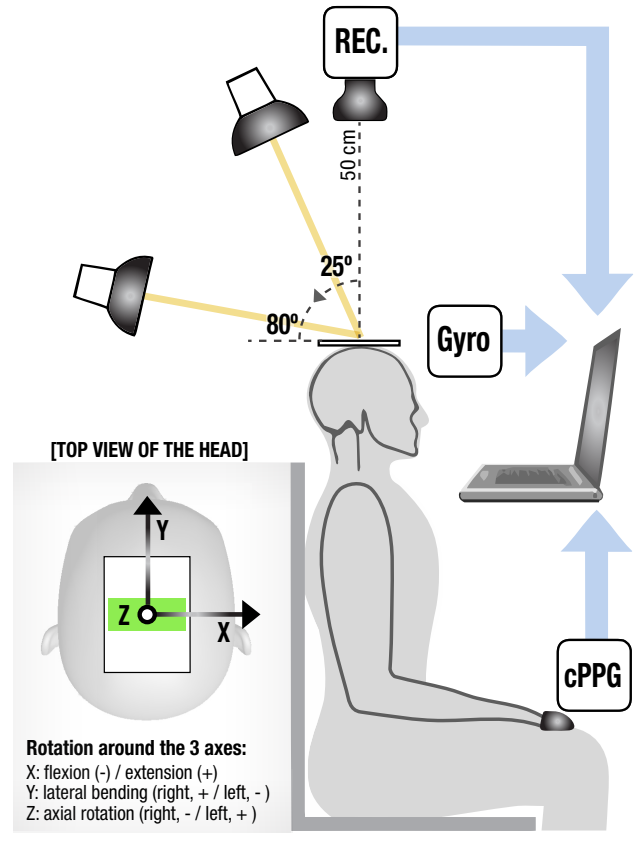

Fig. 2. Schematic view of the experimental setup, with the reference axis for the triaxial gyroscope and the surface to the subject's head. The region of interest within the surface is marked by a green square. Abbreviations: cPPG, contact-PPG by pulse oximetry; Gyro, gyroscope; REC, RGB camera.

MP67B was covered by an opaque, white plastic surface, which was recorded with an RGB camera (model USB UI2230SE-C, IDS, Germany; global shutter, CCD, 8 bits per color channel)(see Fig. 1). To help stabilize this surface within the field-of-view of the camera, the realtime video recordings were shown to the subject in a front screen. Recordings of the surface were acquired in an uncompressed data format (frame rate, $40 \mathrm{~Hz}$; frame size, $384 \times 288$ pixels). The illuminator was an incandescent light source, providing uniform illumination on an RoI of about $2 \mathrm{~cm} \times 7 \mathrm{~cm}(60 \times 210$ pixels $)$. Consecutive recordings of 3 min each were performed for the incident angles of $25^{\circ}$ and $80^{\circ}$.

Signal processing: All signals were processed by standard and custom software implemented in MATLAB. Raw artifact signals were obtained for each recording by averaging red, green and blue pixels within a static RoI on the recorded surfaces. These were lowpass filtered to extract its "DC" component (9th order Butterworth filter; cutoff frequency, 30 $\mathrm{bpm}$ ) and normalized as AC/DC. For the illumination angles of $25^{\circ}$ and $80^{\circ}$, the normalized BCG-artifact signals were denoted as $B C G_{25}$ and $B C G_{80}$, respectively.

Noise removal was the next processing step: motion and artifact signals were processed in strides of 1024 samples (corresponding to about 10 cardiac cycles) with an overlapping factor of $50 \%$. Each stride was detrended, convolved with a Hanning window and filtered in the frequency domain according to an adaptive approach similar to that of [11], where the reference heart-rate was obtained from pulse oximetry signals. For each stride, the frequency bands that exceeded a tolerance width, $\pm \Delta_{n}$, around each of the first $N$ harmonics of the heartrate, $\left[-\Delta_{n}+f_{n}, \Delta_{n}+f_{n}\right]$, were removed, for $n=1 \ldots N$. In our implementation, $N$ was set to 8 harmonics, and $\Delta_{n}=4$ 
bins, except for $\Delta_{1}=2$ bins, as spectra was noisier around $f_{1}$. Then, we obtained average waveforms for motion and artifacts. To this end, the peaks from pulse oximetry signals were used to demarcate cardiac cycles within the data. Individual cycles were resampled to 100 samples and selected according to the following conditions: a) Each relative peak is maximal for the current cycle and also for the neighborhood $[-50,50] \%$ (of the cycle duration) centered at that peak; b) The relative temporal spacing between the peak of the cycle and any of its neighbors is within 80-120\%; and c) The P2P amplitude of the cycle is within the $95 \%$ limits of agreement for the entire signal.

The gyroscope signals were integrated via the trapezoidal method to yield angular displacements, $\theta_{X}(n), \theta_{Y}(n)$ and $\theta_{Z}(n)$. Similarly, the accelerometer signals were integrated twice to yield linear displacements, $d_{X}(n), d_{Y}(n)$ and $d_{Z}(n)$. To estimate the strength of motion and artifacts, we computed the peak-to-peak amplitude of the average waveforms for $B C G_{25}, B C G_{80}$, and displacements.

\section{B. Implications for PPGI at the human face}

Up to now, we considered a small, flat RoI for which incident light rays are parallel. We now move a step further into realistic modeling of BCG-artifacts in PPG-images by considering a topographic representation of a human face. To this end, we used a 3D mesh model randomly chosen from the Basel face model database [23]. For each triangle of the facial representation, the reflected light was computed as the summation of a diffuse Lambertian term and the Torrance-Sparrow analytic bidirectional reflection distribution function. Parameters were extracted from the MERL/ETH Skin Reflectance Database (male adult, skin type II [24]).

To calibrate the model with realistic head rotation and linear displacement parameters, we applied the procedure described in Section II-A3 on a sample 54 subjects (52 male; $25.6 \pm$ 3.7 yrs). The study was approved by the Internal Committee Biomedical Experiments of Philips Research, and an informed consent was obtained from each subject. Angular peak-to-peak displacements (means \pm standard deviations) were dominant for flexion/extension $\left(0.12 \pm 0.03^{\circ}\right)$ and maximal for forward/backward translation $(0.85 \pm 0.30 \mathrm{~mm})^{2}$, so we modeled neck extension/flexion around the $X$ axis by $0.1^{\circ}$ and anterior/posterior displacement by $0.5 \mathrm{~mm}$ (parameter estimated by correcting the accelerometer estimate for linear displacement from rotation; i.e., $0.5 \mathrm{~mm} \approx d_{Z, P 2 P}-\rho \theta_{X, P 2 P}$, with $\rho$ in the range of 15 to $20 \mathrm{~cm}$ ).

To evaluate the effect of a near and distant light source, we set the distance parameter, $R$, to $25 \mathrm{~cm}$ and $1 \mathrm{~m}$, respectively. For each case, we considered three incident angles w.r.t. the baseline position of the face: frontal light, oblique light $\left(45^{\circ}\right.$ w.r.t. normal to the face) and a high angle of incidence (about $90^{\circ}$ w.r.t. normal to the face). For all scenarios, we considered

${ }^{2}$ In this calibration experiment, peak-to-peak angular displacements were $0.12 \pm 0.03^{\circ}, 0.06 \pm 0.02^{\circ}, 0.07 \pm 0.03^{\circ}$ for the $X, Y$ and $Z$ directions, respectively, whereas linear displacements were $0.48 \pm 0.17 \mathrm{~mm}, 0.85 \pm 0.30$ $\mathrm{mm}$ and $0.10 \pm 0.03 \mathrm{~mm}$ for $X, Y$ and $Z$. Flexion/extension is the dominant degree of freedom at the head-neck system because 1 . the common carotid arteries are symmetric w.r.t. the sagittal plane, and 2. both branches are subject to similar cardiac-related pressure variations, so lateral forces cancel out. a frontal camera pointing to the face, at a distance of 50 $\mathrm{cm}$. Then, the intensity at each facet $i$ was computed for the baseline and displaced positions. Using the notation $I_{0}^{(i)}$ and $I_{P 2 P}^{(i)}$, these were computed as follows:

$$
\begin{aligned}
I_{0}^{(i)} & =\frac{I_{0}}{\left\|\mathbf{p}_{0}^{(i)}-\mathbf{p}_{L S}\right\|^{2}} \max \left(0,-\frac{\mathbf{p}_{0}^{(i)}-\mathbf{p}_{L S}}{\left\|\mathbf{p}_{0}^{(i)}-\mathbf{p}_{L S}\right\|} \cdot \hat{\mathbf{e}}_{n, 0}^{(i)}\right) \\
I_{P 2 P}^{(i)} & =\frac{I_{0}}{\left\|\mathbf{p}_{P 2 P}^{(i)}-\mathbf{p}_{L S}\right\|^{2}} \max \left(0,-\frac{\mathbf{p}_{P 2 P}^{(i)}-\mathbf{p}_{L S}}{\left\|\mathbf{p}_{P 2 P}^{(i)}-\mathbf{p}_{L S}\right\|} \cdot \hat{\mathbf{e}}_{n, P 2 P}^{(i)}\right)
\end{aligned}
$$

where: $\hat{\mathbf{e}}_{n, 0}^{(i)}$ is the normal to facet $i$ and $\hat{\mathbf{e}}_{n, P 2 P}^{(i)}=$ $\mathbf{R}_{X}\left(0.11^{o}\right) \hat{\mathbf{e}}_{\mathbf{n}, \mathbf{0}}^{(i)}$ is the corresponding displaced normal; $\mathbf{p}_{L S}=(0,-R \cos \alpha, R \sin \alpha)^{T}$ is the location of the source, for $\alpha$ defined w.r.t. the $Y$ axis; and $\mathbf{p}_{0}^{(i)}$ and $\mathbf{p}_{P 2 P}^{(i)}$ are the baseline and displaced locations of the center of facet $i$, respectively, for $i=1 \ldots 10^{6}$. Finally, the relative intensity of BCG-artifacts at the face representation, $Y_{f}^{(i)}$, was computed for each facet $i$ and expressed as the ratio "AC" over "DC". This expression was approximated by $\left|I_{0}^{(i)}-I_{P 2 P}^{(i)}\right| / I_{0}^{(i)}$, if $I_{0}^{(i)}$ is positive, or 0 otherwise. To prevent numerical instability, $Y_{f}^{(i)}$ was truncated to $1 \%$.

\section{Proof-of-concept on real skin}

While the model-based approach conducted so far allows a solid understanding of BCG effects, no results were generated that reflect pollution of signals on actual skin. This is why we conducted an additional experiment with different conditions of face illumination meant to allow qualitative contrast between minimized versus boosted BCG-artifacts at the forehead. Although it is not possible to isolate BCG from PPG, by having skin and non-skin RoIs placed next to each other, at least a reference waveform can be measured for this artifact. So we used black tape and white plastic surfaces to demarcate pairs of rectangular RoIs within the subject's forehead. For each pair, one RoI consisted of skin and another consisted of white opaque plastic board (sizes 2 $\mathrm{cm} \times 3-3.5 \mathrm{~cm}$; pixels resolution in the order of 50 pixels $^{2}$ ). For demonstrative purposes, one pair of RoIs is sufficient to investigate effects of non-uniform lighting over the strength of BCG-artifacts, so we chose, arbitrarily, the middle one (see Fig. 3). Data acquisition and signal processing are described in sections II-C1 and II-C2, respectively.

1) Data acquisition: The data acquisition protocol of Appendix II-A3 was adapted for measurements of regions of interest at the forehead on the same male subject as in II-A3. The previously described RGB camera and pulse oximeter were reused (sampling frequency, $20 \mathrm{~Hz}$ ), and the subject was required to sit on a chair while avoiding voluntary motions. However, the fluorescent lamp Philips EnergyLight (reference HF3319) was now preferred, owing to the provision of diffuse illumination. Also, this source has spectral spikes at $439 \mathrm{~nm}$, $550 \mathrm{~nm}$ and $615 \mathrm{~nm}$, a feature that minimizes inter-channel interference when Bayer filters are used.

Two consecutive recordings of 2 min were undertaken. First, non-uniform lighting conditions were achieved by placing a 
single fluorescent lamp (FL) about $30 \mathrm{~cm}$ over the subject's head, with the light pointing downwards (see FL 1 at Fig. 3(a)). Then, three additional fluorescent lamps were included in the setup to provide uniform illumination at the forehead: one positioned above the head, to radiate light upwards (FL $4)$, and the remaining ones positioned near the camera and oriented frontwards to the subject, at a distance of about $50 \mathrm{~cm}$ (FL 2,3). Care was taken to minimize specular reflections by covering the camera lens and all lamps with linear polarizing film. Figures 3(b) and 3(c) show sample frames obtained under top and uniform illumination, respectively.

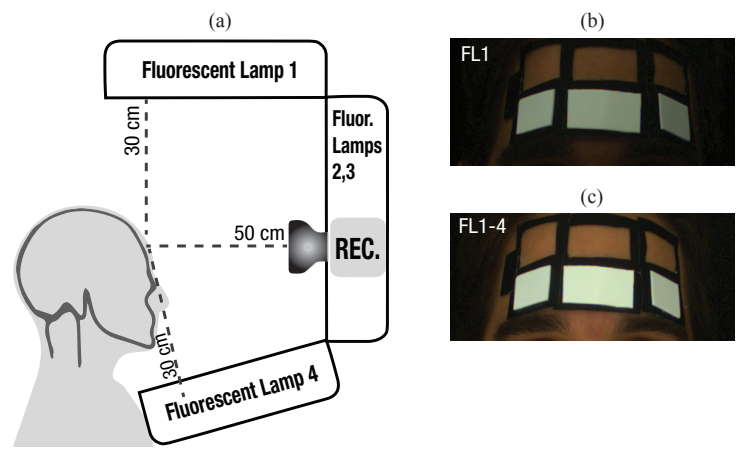

Fig. 3. Setup for data acquisition at the forehead of a seated subject, including an RGB camera and fluorescent lamps (FL; top lighting conditions requires FL 1 whereas, for uniform illumination, FL 1-4 are used).

2) Signal processing: The temporally varying remote-PPG signals were extracted from skin and non-skin RoIs at the forehead by averaging the corresponding pixels, tracked over time. We processed signals separately for the R, G and B channels in skin and jointly for the non-skin surface. Similar to Section II-A3, all strides were normalized as AC/DC to allow comparison of signals irrespective of camera aperture, local brightness and albedo of surface, whether it is skin or non-skin (e.g., the white plastic surface is brighter than the skin RoI). Additionally, the AC/DC representation promotes invariance to lighting variations and reduces low-frequency noise [25]. Adaptive bandpass-filtering was applied around the pulse-rate frequency plus the first 3 harmonics using the shorttime Fourier transform (window size, 128; overlap-and-add by a factor of $50 \%$, with a Hanning window). Then, the peaks from pulse oximetry were used to demarcate cardiac cycles in the signals from the skin and non-skin RoIs. These were resampled to 24 samples per cycle and averaged to obtain representative PPG and BCG waveforms.

\section{RESULTS}

Table I contrasts peak-to-peak angular displacements and artifacts that were determined-experimentally, according to section II-A3, and as predicted from (10) - for the incident angles, $\alpha$, of $25^{\circ}$ and $80^{\circ}$ (i.e., near-frontal vs. near-lateral lighting conditions). For similar displacements, the strength of artifacts seems to differ by almost an order of magnitude.

Fig. 4 depicts simulation results for motion maps obtained under contrasting distances to source $(R=25 \mathrm{~cm}$ and 100 $\mathrm{cm}$ ) and incident angles (top light; $45^{\circ}$ w.r.t. the normal to the face and frontal light). Overall, artifacts are strongest when the
TABLE I

BCG-MOTION AND ARTIFACTS UNDER NON-UNIFORM ILLUMINATION.

\begin{tabular}{|c|ccc|cc|}
\hline$\alpha$ & $\theta_{X, P 2 P}$ & $\theta_{Y, P 2 P}$ & $\theta_{Z, P 2 P}$ & Experimental & From (10) \\
\hline $25^{\circ}$ & $0.23^{\circ}$ & $0.06^{\circ}$ & $0.11^{\circ}$ & $0.26 \%$ & $0.17 \%$ \\
$80^{\circ}$ & $0.21^{\circ}$ & $0.08^{\circ}$ & $0.11^{\circ}$ & $1.86 \%$ & $2.27 \%$ \\
\hline
\end{tabular}

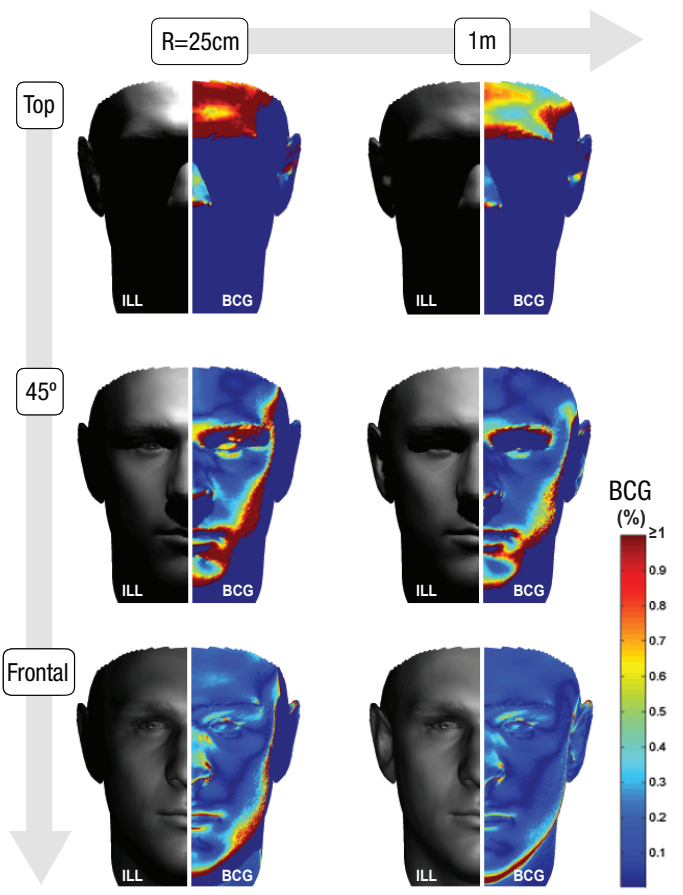

Fig. 4. Amplitude of BCG-artifacts at a human face, expressed in normalized percentage. Simulations were carried for different incident angles (frontal, $45^{\circ}$ w.r.t. the normal-to-face and top light) and distance to source $(25$ and $100 \mathrm{~cm}$ ). The right side half-faces illustrate illumination conditions at the face.

incident light largely deviates from orthogonality to surface (particularly in the range of $45^{\circ}$ to $90^{\circ}$ ), an issue promoted by shorter source-to-face distances (e.g., $R=25 \mathrm{~cm}$ ).

Finally, Fig. 5 shows the resulting PPG and BCG waveforms obtained on skin and non-skin adjacent surfaces at the forehead, under non-uniform and uniform lighting conditions. A striking observation from this proof-of-concept experiment is the fact that artifacts are clearly most prominent under top lighting and are particularly detrimental to the smaller amplitude PPG signals acquired at the red and blue channels. By inspecting the frequency domain representations under top light (see Fig. 5(b)), one can further verify that the energy of BCG-artifacts is mostly at the second and third harmonics of the cardiac frequency (respectively 0.0087 and 0.130 in a normalized frequency range from $0-1$, where 1 corresponds to sampling frequency). Conversely, remote PPG signals are low frequency signals whose energy is mostly concentrated at the fundamental pulse-rate frequency. Homogenous lighting improved waveform shapes in all channels, although there seems to be remnants of BCG-artifacts at the blue wavelength.

\section{DISCUSSION}

This paper considers the effect of ballistocardiographic artifacts in remote PPG-images. The overall procedure taken in 

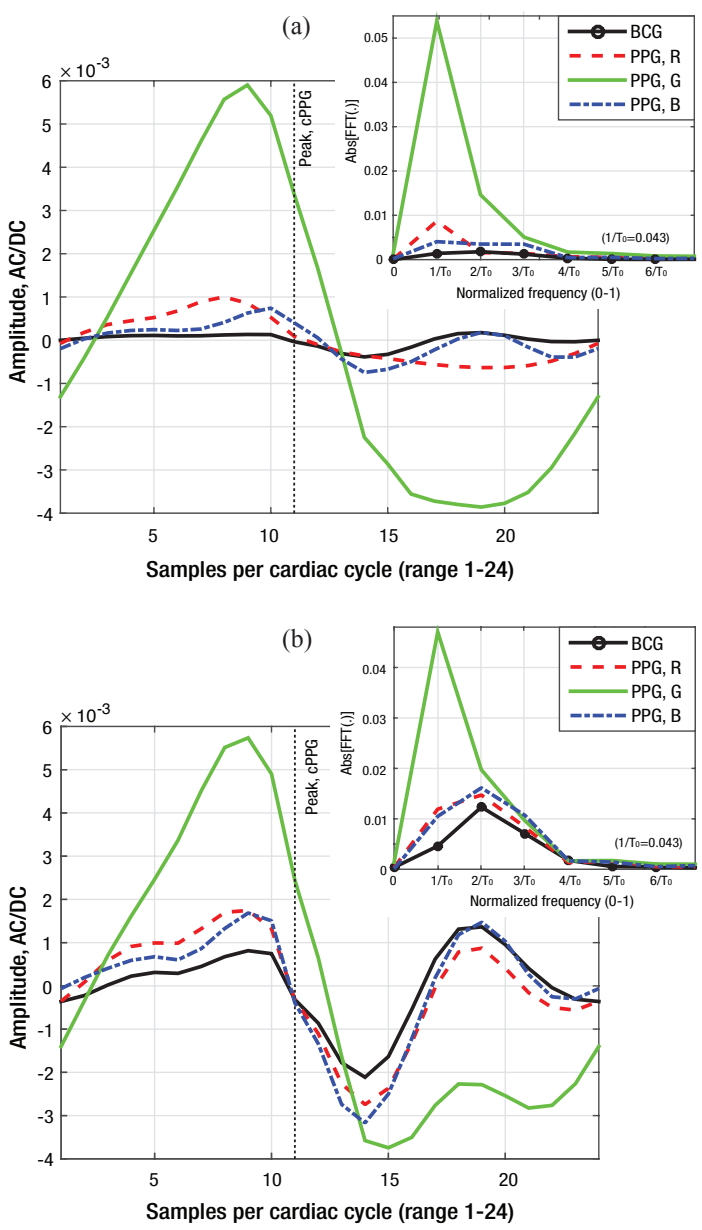

Fig. 5. Remote PPG and artifact signals obtained under (a) homogenous and (b) non-homogenous lighting) conditions. The dotted vertical line at time representations denotes the peak of the [reference] pulse oximetry signal.

this investigation consisted of three steps: 1 . building a model for BCG-artifacts at the head with realistic estimates of head motion, 2. applying the model to study the effect of nonuniform illumination on BCG-artifact strength at the human face, and 3. demonstrating actual BCG effects on real skin.

To allow comparison of the relative strength of BCG vs. PPG signals (\%), Fig. 6 provides a joint representation of relative BCG-artifacts and PPG signals. Specifically, Fig. 6(a) illustrates the peak-to-peak amplitude of BCG signals and its derivative, as a function of angle of incidence, $\alpha$, for neck flexion/extension, $\theta_{X}$, set to $0.1^{\circ}$. It is apparent that the amplitude of BCG-artifacts increases slowly until $45^{\circ}$ but rises sharply for $\alpha>45^{\circ}$. As practical implication, artifacts are minimized under orthogonal lighting conditions. However, the reverse situation occurs for lateral illumination. These expectations were confirmed in the validation experiment reported in Section II-A3, where the average BCG waveform was measured for the contrasting scenarios of $\alpha$ set to $25^{\circ}$ (near-frontal) and $80^{\circ}$ (near-lateral). Despite contamination by various noisy sources (e.g., model error, muscular tremor, specular reflections, thermal noise from the sensors, drift of the subject's head w.r.t. baseline position, affecting the accuracy of $\alpha$, etc.), the amplitude of the experimentally measured BCG-

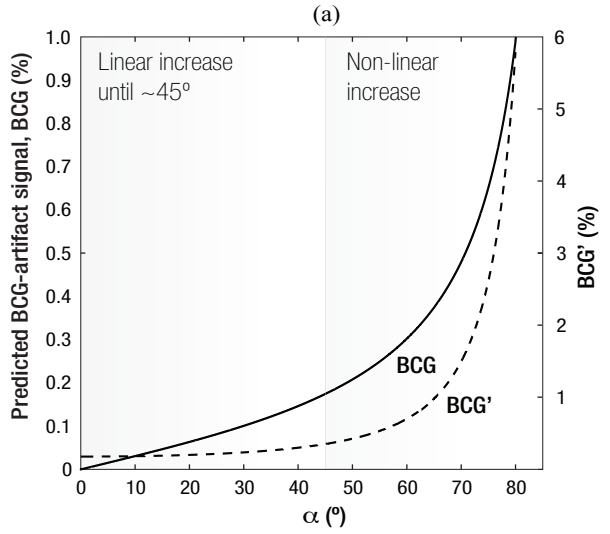

(b)

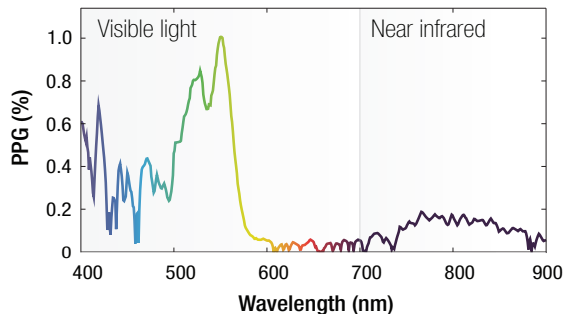

Fig. 6. (a) Relative BCG artifact and its derivative as a function of the angle between incident light and normal to surface, $\alpha$, for $\theta$ set equal to $0.1^{\circ}$. (b) The relative PPG-spectrum by van Gastel [6] from the measurements of the absolute spectrum by Corral et al. [26]. The spectrum was scaled to $1 \%$ for its peak location, which is a realistic value but varies over individuals [4]

artifacts (for $\alpha$ set to $25^{\circ}$ and $80^{\circ}$ ) is close to what would be expected from (10) (see Table I). Note that BCG-artifacts are sensitive to the tangent of the incident angle of light, $\alpha$, and to the peak-to-peak angular displacement of the head, $\theta_{X}$, but are wavelength independent; this property is valid irrespective of the spectral characteristics of the illuminator.

To put the amplitude of BCG-artifacts into perspective, Fig. 6(b) depicts the relative amplitude of remote-PPG signals as a function of wavelength, as derived from the PPG spectrum measurements of Corral et al. [26], [6], where the maximal signal strength was scaled to $1 \%$ [4]. This spectrum has two limitations; first, its shape suffers from measurement noise, in particular for shorter wavelengths and, second, the head was not fixed during data acquisition [26], thus affecting precision and accuracy of measurements. Still, the existing data is already valuable to show that the strength of PPG signals is wavelength-dependent and peaks at the green region of the spectrum. Most importantly, the pairwise representation of the relative BCG (as a function of angle of incidence) and PPG signals (as a function of wavelength) allows one to suspect that the relative BCG-artifacts can be comparable or stronger than actual PPG signals, particularly in non-green wavelengths and under non-orthogonal angles of incidence. As the accurate amplitude of signals is mandatory for mapping the spatial distribution of blood-volume variations in the skin, nonuniform lighting and pulse-synchronous motion are, indeed, serious concerns to the existing PPG imaging systems.

The motion maps obtained using a mesh model of the human face (Fig. 4) suggest that distant and frontal sources cause the least BCG-artifacts at most regions of the face. For 
the more distant light source, the same extent of head motion results in lower variations of the radiant light flux in a given RoI. Hence, preferring distant light sources (about $1 \mathrm{~m}$ ) is a means to minimize BCG-artifacts. This option provides the added benefit of homogenous illumination, as was previously pointed out by Rubins et al. [8].

Finally, in a proof-of-concept experiment on real skin vs. non-skin (Fig. 5), artifacts are demonstrated under nonuniform lighting conditions. These are particularly detrimental if acquisition occurs at wavelengths where PPG signals are typically low-amplitude; i.e., the red and blue channels (Fig. 6(b)). On the bright side, this experiment also shows that this problem can be minimized under orthogonal lighting conditions. We managed to achieve this goal in a simple manner by placing additional diffused light sources around the forehead RoIs to be inspected, although we recognize that further work is needed to improve performance at the blue region of the spectrum. Because penetration depth is shallowest for blue, this wavelength is most sensitive to contamination and non-ideal experimental conditions, including lighting and leaking specular reflections (not fully removed by linear polarizers). Still, the PPG strength in the blue channel may be obtained by correlation with the green or red as reference waveforms.

As far as peak-to-peak amplitude is concerned, the PPG waveform obtained at the green channel did not suffer significantly, primarily because the strength of PPG signals at the $550 \mathrm{~nm}$ green light peak is much stronger than the artifact for most incident angles. Since our light source is diffuse and allows incident angles up to maximally $80^{\circ}$, the artifacts in this example for green are modest. Still, even in this nonextreme situation, artifacts are still dominant in the diastolic phase of the cardiac cycle. One may expect the scenario to be much worse when roof illumination or punctual light sources are used, as incident angles may easily go well above $45^{\circ}$. Zheng et al. [27] is a flagrant example. Hereby, the authors describe a PPG-imager meant to capture PPG waveforms from he human face at red and infrared wavelengths (650 and 870 $\mathrm{nm}$, respectively). At the core of this innovation is a ring unit consisting of multiple light emitting diodes (LEDs) with a parabolic reflector, which is mounted around the camera lens. This structure is claimed to provide homogeneous illumination at the human face. However, the experimental setup indicates that the forehead is illuminated by light hitting mostly by incident angles $>80^{\circ}$, so artifacts may arise in the outcome images. These suspicions are confirmed by the thus-obtained Fourier representation of the remote PPG signals, particularly at the $650 \mathrm{~nm}$ wavelength, as the second and third order harmonics are stronger than the fundamental component.

Efforts to achieve homogenous illumination using external ringlights have also been attempted by Wieringa et al.[28]. PPG-images of a wrist were obtained in frontal illumination conditions, with LEDs of red and infrared wavelengths placed around the camera. However, the relatively higher spectral power at the edges of this wrist was not explained by the authors, nor seems to have a physiological explanation. Instead, these are the most likely artifacts, as the angle between LEDs and skin-normal is highest at the edges of the wrist, an hypothesis that is corroborated the explanation of Blanik et al. [20] about a similar finding in their own wrist data. The wrist was further mapped by Kamshilin et al. [29], using two green LEDs placed around the camera. Although illumination was assumed to be almost uniform, the schematic view of the setup for video recordings suggests it to be non-uniform, with angles of incidence between LEDs and skin-normal that can exceed $45^{\circ}$. This illumination condition raises doubts about interpretations derived from the resulting amplitude or phase maps, particularly at the locations of stronger arterial motion, as are the radial and ulnar arteries. It seems, therefore, plausible, that the reported hotspots are just skin sites where BCG-artifacts are maximal. Additional examples of artifacts in edges may also be found in the skin boundaries of the hand or fingers [11], [8] and the forehead of a seated subject [5].

Future work could be aimed at improving sensing modalities for estimating and adaptively canceling artifacts from actual PPG signals. In this regard, a better understanding of micromotion in different skin sites, positions and populations would be valuable. For example, Dziuda et al. [30] found differences in BCG signals for the standing and seating positions using a fiber-optic technology, whereas March et al. [31] found aging to have a significant effect on the BCG amplitude.

\section{CONCLUSION}

We demonstrated the threat of BCG-artifacts on PPGimagers under non-uniform lighting conditions. These artifacts add to PPG sensors by modulating the angle between skin surface and incident light, thus distorting the resulting waveform at the PPG-image sensor array. From our calibrated model for head motion and additional experiment at the forehead, we were able to show that BCG-artifacts are most detrimental under incident angles approaching $90^{\circ}$ w.r.t. the surfacenormal. In addition, we have indicated various publications which suffered from these artifacts. We proposed to ameliorate this problem during data acquisition by preferring orthogonal illumination, a recommendation whose efficacy is not affected by the location of the camera and is simply achieved, for example, by preferring distant illumination and/or by deploying several diffused light sources around the skin region-ofinterest. We hope that our paper can help prevent erroneous interpretations of PPG-images in future publications.

\section{ACKNOWLEDGMENT}

The authors are grateful to Vincent Jeanne and Dr. I. Kirenko from Philips Research for their support with the materials, as well as to Benoit Balmaekers for assisting in motion measurements. For helpful discussions, we thank Dr. W. Verkruysse, Mark van Gastel and Wenjin Wang. Thanks also to all the volunteers who participated in this study. This research was performed within the framework of the IMPULS-2 program on Advanced Peri-Operative Monitoring.

\section{REFERENCES}

[1] J. Allen, "Photoplethysmography and its application in clinical physiological measurement," Physiol. Meas., vol. 28, no. 3, pp. 1-40, 2007. 
[2] M. Huelsbusch and V. Blazek, "Contactless mapping of rhythmical phenomena in tissue perfusion using PPGi," Medical Imaging 2002: Physiology and Function from Multidimensional Images, vol. 4683, pp. 110-117, 2002.

[3] V. Blazek, T. Wu, and D. Hoelscher, "Near-infrared CCD imaging: possibilities for noninvasive and contactless $2 \mathrm{D}$ mapping of dermal venous hemodynamics," in Proc. SPIE, vol. 3923, 2014, pp. 2-9.

[4] W. Verkruysse, L.O. Svaasand, and J.S. Nelson, "Remote plethysmographic imaging using ambient light," Opt. Express, vol. 16, no. 26, pp. $21434-21445,2008$.

[5] N. Blanik, A.K. Abbas, B. Venema, V. Blazek, and S. Leonhardt, "Hybrid optical imaging technology for long-term remote monitoring of skin perfusion and temperature behavior," J. Biomed. Opt., vol. 19 no. 1, p. 16012, 2014

[6] M. van Gastel, S. Stuijk, and G. de Haan, "Motion robust remote-PPG in infrared," IEEE Trans. Biomed. Eng., vol. PP, no. 99, pp. 1-9, 2015.

[7] J. Allen and K. Howell, "Microvascular imaging: techniques and opportunities for clinical physiological measurements," Physiol. Meas., vol. 35, no. 7, p. R91, 2014.

[8] U. Rubins, V. Upmalis, O. Rubenis, D. Jakovels, and J. Spigulis, "Real-time photoplethysmography imaging system," in 15th NordicBaltic Conf. Biomed. Eng. and Medical Physics, ser. Proc. IFMBE, K. Dremstrup, S. Rees, and M. Jensen, Eds., vol. 34, 2011, pp. 183-186.

[9] J. Spigulis, "Biophotonic technologies for non-invasive assessment of skin condition and blood microcirculation," p. 63, 2012.

[10] L. Feng, L.M. Po, X. Xu, Y. Li, and R. Ma, "Motion-resistant remote imaging photoplethysmography based on the optical properties of skin," IEEE Trans. Circuits Syst. Video Technol., vol. PP, no. 99, 2014.

[11] A.A. Kamshilin, S. Miridonov, V. Teplov, R. Saarenheimo, and E. Nippolainen, "Photoplethysmographic imaging of high spatial resolution," Opt. Express, vol. 2, no. 4, pp. 996-1006, 2011.

[12] S. de Ridder, P.F. Migeotte, X. Neyt, N. Pattyn, and G.K. Prisk, "Threedimensional ballistocardiography in microgravity: A review of past research," in Ann. Int. Conf. of the IEEE Engineering in Medicine and Biology Society (EMBC), 2011, pp. 4267-4270.

[13] O. Inan, M. Etemadi, R.M. Wiard, L. Giovangrandi, and G.T.A. Kovacs, "Robust ballistocardiographic acquisition for home monitoring," Physiol. Meas., vol. 30, no. 9, pp. 169-185, 2009.

[14] L. Dziuda, "Fiber-optic sensors for monitoring patient physiological parameters: a review of applicable technologies and relevance to use during magnetic resonance imaging procedures," J. Biomed. Opt., vol. 20, no. 1, p. 010901, 2015.

[15] I. Starr, A.J. Rawson, H.A. Schroeder, and N.R. Joseph, "Studies on the estimation of cardiac output in man, and of abnormalities in cardiac function, from the heart's recoil and the blood's impacts; the ballistocardiogram," Am. J. Physiol., vol. 127, no. 1, pp. 1-28, 1939.

[16] D.D. He, E.S. Winokur, and C.G. Sodini, "A continuous, wearable, and wireless heart monitor using head ballistocardiogram (BCG) and head electrocardiogram (ECG)," in 33rd Int. Conf. IEEE EMBS, 2011.

[17] J. Hernandez, Y. Li, J.M. Rehg, and R.W. Picard, "Bioglass: Physiological parameter estimation using a head-mounted wearable device," in Int. Conf. on Wireless Mobile Communication and Healthcare, 2014.

[18] G. Balakrishnan, F. Durand, and J. Guttag, "Detecting pulse from head motions in video," in IEEE Conf. on Computer Vision and Pattern Recognition (CVPR), 2013, pp. 3430-3437.

[19] P. LeVan, J. Maclaren, M. Herbst, R. Sostheim, M. Zaitsev, and J. Hennig, "Ballistocardiographic artifact removal from simultaneous EEGfMRI using an optical motion-tracking system," Neurolmage, vol. 75, pp. 1-11, 2013

[20] N. Blanik, C. Blazek, C. Pereira, V. Blazek, and S. Leonhardt, "Frequency-selective quantification of skin perfusion behavior during allergic testing using photoplethysmography imaging," in Proc. SPIE, vol. 9034, 2014, pp. 903 429-903 429-6.

[21] A. Stewart, M. Marfell-Jones, and The International Society for the Advancement of Kinanthropometry (ISAK), International Standards for Anthropometric Assessment. ISAK, 2006.

[22] M. Simoneau, M. Denninger, and T.C. Hain, "Role of loading on head stability and effective neck stiffness and viscosity," J. Biomech., vol. 41, no. 10, pp. 2097-2103, 2008.

[23] P. Paysan, R. Knothe, B. Amberg, S. Romdhani, and T. Vetter, "A 3D face model for pose and illumination invariant face recognition," in 6th IEEE Conf. on Advanced Video and Signal Based Surveillance, 2009.

[24] T. Weyrich, W. Matusik, H. Pfister, B. Bickel, C. Donner, C. Tu, J. McAndless, J. Lee, A. Ngan, H.W. Jensen, and M. Gross, "Analysis of human faces using a measurement-based skin reflectance model," $A C M$ Trans. Graph., vol. 25, no. 3, pp. 1013-1024, Jul. 2006.
[25] G. de Haan and Jeanne, V., "Robust pulse-rate from chrominance-based rPPG," IEEE Trans. Biomed. Eng., 2014.

[26] L.F. Corral, G. Paez, and M. Strojnik, "Optimal wavelength selection for non-contact reflection photoplethysmography," 22nd Congress of the International Commission for Optics: Light for the Development of the World, vol. 8011, 2011.

[27] J. Zheng, S. Hu, A.S. Echiadis, V. Azorin-Peris, P. Shi, and V. Chouliaras, "A remote approach to measure blood perfusion from the human face," in Proc. SPIE, vol. 7169, 2009, pp. 171-177.

[28] F.P. Wieringa, F. Mastik, and A.F.W. van der Steen, "Contactless multiple wavelength photoplethysmographic imaging: A first step toward "SpO2 camera" technology," Ann. Biomed. Eng., vol. 33, no. 8, 2005.

[29] A.A. Kamshilin, E. Nippolainen, I.S. Sidorov, P.V. Vasilev, N.P. Erofeev, N.P. Podolian, and R.V. Romashko, "A new look at the essence of the imaging photoplethysmography," Sci. Rep., vol. 5, 2015.

[30] L. Dziuda and F.W. Skibniewski, "A new approach to ballistocardiographic measurements using fibre Bragg grating-based sensors," Biocybern. Biomed. Eng., vol. 34, no. 2, pp. 101 - 116, 2014.

[31] H.W. March, "Three-plane ballistocardiography: The effect of age on the longitudinal, lateral, and dorsoventral ballistocardiograms," Circulation, vol. 12, no. 5, pp. 869-882, 1955 .

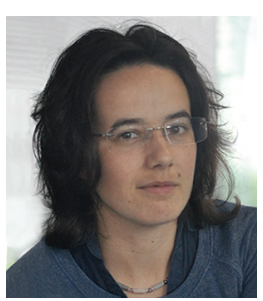

Andreia V. Moço received M.Sc degrees in electronics and telecommunications engineering (2008) and exercise and health (2014), from Aveiro and Lisbon Universities, respectively (Portugal). She is currently a $\mathrm{PhD}$ candidate in the Department of Electrical Engineering at Eindhoven University of Technology in cooperation with Philips Research Eindhoven. Her research interests include signal processing, computer vision and remote-PPG imaging.

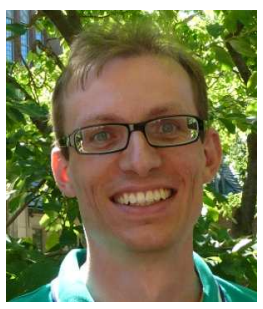

Sander Stuijk received his M.Sc. (with honors) in 2002 and his Ph.D. in 2007 from the Eindhoven University of Technology. He is currently an assistant professor in the Department of Electrical Engineering at Eindhoven University of Technology. He is also a visiting researcher at Philips Research Eindhoven working on bio-signal processing algorithms and their embedded implementations. His research focuses on modeling methods and mapping techniques for the design and synthesis of predictable systems with a particular interest into bio-signals.

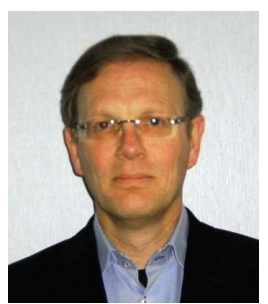

Gerard de Haan received $\mathrm{BSc}$, $\mathrm{MSc}$, and $\mathrm{PhD}$ degrees from Delft University of Technology in 1977, 1979 and 1992, respectively. He joined Philips Research in 1979 to lead research projects in the area of video processing/analysis. From 1988 till 2007, he has additionally taught post-academic courses for the Philips Centre for Technical Training at various locations in Europe, Asia and the US. In 2000, he was appointed "Fellow" in the Video Processing \& Analysis group of Philips Research Eindhoven, and "Full-Professor" at Eindhoven University of Technology. He has a particular interest in algorithms for motion estimation, video format conversion, image sequence analysis and video health monitoring His work in these areas has resulted in 4 books, 3 book chapters, 180 scientific papers and more than 180 patent applications, and various commercially available ICs. He received 5 Best Paper Awards, the Gilles Holst Award, the IEEE Chester Sall Award, bronze, silver and gold patent medals, while his work on motion received the EISA European Video Innovation Award, and the Wall Street Journal Business Innovation Award. Gerard de Haan serves in the program committees of various international conferences on image/video processing and analysis, and has been a Guest-Editor for special issues of Elsevier, IEEE, and Springer. 